\title{
WORK-COMPETITIVE SCHEDULING FOR COOPERATIVE COMPUTING WITH DYNAMIC GROUPS*
}

\author{
CHRYSSIS GEORGIOU ${ }^{\dagger}$, ALEXANDER RUSSELL ${ }^{\ddagger}$, AND ALEXANDER A. SHVARTSMAN $\S$
}

\begin{abstract}
The problem of cooperatively performing a set of $t$ tasks in a decentralized computing environment subject to failures is one of the fundamental problems in distributed computing. The setting with partitionable networks is especially challenging, as algorithmic solutions must accommodate the possibility that groups of processors become disconnected (and, perhaps, reconnected) during the computation. The efficiency of task-performing algorithms is often assessed in terms of work: the total number of tasks, counting multiplicities, performed by all of the processors during the computation. In general, the scenario where the processors are partitioned into $g$ disconnected components causes any task-performing algorithm to have work $\Omega(t \cdot g)$ even if each group of processors performs no more than the optimal number of $\Theta(t)$ tasks.

Given that such pessimistic lower bounds apply to any scheduling algorithm, we pursue a competitive analysis. Specifically, this paper studies a simple randomized scheduling algorithm for $p$ asynchronous processors, connected by a dynamically changing communication medium, to complete $t$ known tasks. The performance of this algorithm is compared against that of an omniscient off-line algorithm with full knowledge of the future changes in the communication medium. The paper describes a notion of computation width, which associates a natural number with a history of changes in the communication medium, and shows both upper and lower bounds on work-competitiveness in terms of this quantity. Specifically, it is shown that the simple randomized algorithm obtains the competitive ratio $(1+\mathbf{c w} / e)$, where $\mathbf{c w}$ is the computation width and $e$ is the base of the natural logarithm $(e=2.7182 \ldots)$; this competitive ratio is then shown to be tight.
\end{abstract}

Key words. on-line algorithms, competitive analysis, partitionable networks, distributed computation, independent tasks, randomized algorithms, work complexity

AMS subject classifications. 68W15, 68W20,68W40, 68Q25, 68Q85

DOI. $10.1137 /$ S0097539704440442

1. Introduction. The problem of cooperatively performing a known set of tasks in a decentralized computing environment subject to failures is one of the fundamental problems in distributed computing. Variations on this problem have been studied in a variety of different settings, including, for example, message-passing models [7, 8, $11]$, shared-memory models $[18,17,2,21,19]$, and partitionable network models [10, 20]. In the settings where network partitions may interfere with the progress of computation, the challenge is to maintain efficiency despite dynamically changing processor connectivity.

This problem is normally abstracted in terms of a set of $t$ tasks that must be performed in a distributed environment consisting of $p$ processors, subject to processor failures and communication disruptions. Algorithmic solutions for this problem are

${ }^{*}$ Received by the editors February 2, 2004; accepted for publication (in revised form) December 8, 2004; published electronically May 12, 2005. This research was supported in part by NSF grant 0311368.

http://www.siam.org/journals/sicomp/34-4/44044.html

†Department of Computer Science, University of Cyprus, Nicosia, Cyprus (chryssis@ucy.ac.cy). This work was performed in part while this author was at the University of Connecticut.

${ }^{\ddagger}$ Department of Computer Science and Engineering, University of Connecticut, Storrs, CT 06269 (acr@cse.uconn.edu). The work of this author was supported in part by NSF Career Award 0093065 and by NSF grants 0220264 and 0218443.

$\S$ Department of Computer Science and Engineering, University of Connecticut, Storrs, CT 06269, and Laboratory for Computer Science and Artificial Intelligence, Massachusetts Institute of Technology, Cambridge, MA 02139 (alex@theory.csail.mit.edu). The work of this author was supported in part by NSF Career Award 9984778 and by NSF grants 9988304 and 0121277. 
typically evaluated by bounding their worst-case work: the total number of computation steps performed by all processors during the computation. We consider the situation where the tasks are similar, that is, completion of each task requires the same number of computation steps, and where task-oriented work dominates local bookkeeping. In this case the work incurred by an algorithm is simply the total number of tasks, counting multiplicities, completed by the processors.

The details of the computation model naturally have a dramatic impact on the existence of efficient (or even interesting) algorithms for the problem. In this paper, we consider the partitionable network scenario consisting of $p$ asynchronous processors with a communication medium that is subject to arbitrary partitions during the life of the computation. This model is motivated by the abstraction provided by a typical group communication scheme; see, for example, the surveys in [23]. Specifically, at each point of the computation, the communication medium effectively partitions the processors into nonoverlapping groups: communication within a group is instantaneous and reliable, communication across groups is impossible. Naturally, processors in the same group can share their knowledge of completed tasks and, while they remain connected, avoid doing redundant work. For the remainder of the paper we refer to a transition from one network partition to another as a reconfiguration.

We do not charge for coordination within a group, simply treating grouped processors as a single (virtual) asynchronous processor. In particular, if a group of processors performs a set of $t$ tasks during the lifetime of that group, we charge this group $t$ units of work, ignoring, for example, partially completed tasks which may remain at the group's demise or the cost of synchronizing processors' knowledge during the group's inception. Each processor may cease executing tasks only when it knows the results of all tasks. While processors are asynchronous, they do not crash.

An algorithm in this model is a rule which, given a group of processors and a set of tasks known by this group to be completed, determines a task for the group to complete next. In the case where all processors are disconnected during the entire computation, any algorithm must incur $\Omega(t \cdot p)$ work. On the other hand, any reasonable algorithm should attain $O(t)$ work in the case where all processors remain connected during the computation. Considering that every algorithm performs poorly in the totally disconnected case, it seems reasonable to treat the problem as an on-line problem and pursue competitive analysis.

Fix, for the moment, an algorithm $A$. For expository purposes, let us treat both the processors' asynchrony and the dynamics of the network as if they were determined by an adversary $\mathcal{A}$. The adversary determines an initial partition $\mathcal{P}_{1}$ of the processors into groups and determines how many tasks each group of this partition $\mathcal{P}_{1}$ completes before the next reconfiguration; while the number of tasks completed by each group is determined by the adversary, the actual subset of tasks (that is, the identity of the tasks) completed by each group is determined by the algorithm $A$. The adversary then determines a reconfiguration of the processors, giving rise to a new partition $\mathcal{P}_{2}$, and, as before, determines how many tasks each of the newly created groups of $\mathcal{P}_{2}$ completes before the next reconfiguration. Any group created during such a reconfiguration is assumed to have the combined knowledge of all its members: any task known to be completed by a processor of the group $G$ is known to be completed by all processors of $G$. This process of reconfiguration and computation continues until every processor is aware of the outcome of every task. Groups with knowledge of the outcome of all tasks cause no work: in effect, they may "idle" until the next reconfiguration. Note that for this algorithm $A$, the work caused by the 
adversary $\mathcal{A}$ is completely determined by (i) the collection of groups that existed during the computation, (ii) the number of tasks $\mathcal{A}$ permits each group to perform, and (iii) for each group $G$, the identities of all those groups in which processors of $G$ have previously been members. (Note that the initial knowledge of the group $G$ is determined in part by (iii).) These characteristics can be captured by a certain directed acyclic graph, to which we refer as a computation pattern. This is formally defined in the next section. Note that different sequences of reconfigurations can in fact give rise to the same computation pattern.

As an example, consider the scenario with 3 processors which, starting from isolation, are permitted to proceed synchronously until each has completed $t / 2$ tasks; at this point an adversary chooses a pair of processors to merge into a group. It is easy to show that if $T_{1}, T_{2}$, and $T_{3}$ are subsets of $[t]$ of size $t / 2$, then there is a pair $\left(T_{i}, T_{j}\right)$ (where $i \neq j$ ) so that $\left|T_{i} \cap T_{j}\right| \geq t / 6$ : in particular, for any scheduling algorithm, there is a pair of processors which, if merged at this point, will have $t / 6$ duplicated tasks; this pair alone must then expend $t+t / 6$ work to complete all $t$ tasks. The optimal off-line algorithm that schedules tasks with full knowledge of future merges, of course, accrues only $t$ work for the merged pair, as it can arrange for zero overlap. Furthermore, if the adversary partitions the two merged processors immediately after the merge (after allowing the processors to exchanged information about task executions), then the work performed by the merged and then partitioned pair is $t+t / 3$; the work performed by the optimal algorithm remains unchanged, since it terminates at the merge.

Contributions. We study upper and lower bounds on the competitiveness of scheduling algorithms for the task-performing problem in partitionable networks. We analyze the natural randomized algorithm for $p$ processors and $t$ tasks, called RANDOM SELECT (RS), in which each processor (or group) determines the next task to complete by randomly selecting the task from the subset of tasks this group does not know to be completed. We compare the expected work of this algorithm to the work of an optimal off-line algorithm, which may schedule tasks with full knowledge of future partitions.

In order to precisely state the results of the paper, we pause to introduce some notation. In the literature, groups of processors are given structured names, such that a group $G$ is a pair $\langle G . i d, G$.set $\rangle$, where $G$.id is the unique identifier of $G$ and G.set is the set of processor identifiers in $[p]$ that determine the members of the group. To reduce notational clutter, given a group named $G$, we use $G$ to stand for $G$.set in this paper (e.g., if two, possibly distinct, groups $G$ and $G^{\prime}$ have identical membership, we express this by $G=G^{\prime}$ ).

As discussed previously, an adversary determines a computation pattern $C$ in a natural way; this is a directed acyclic graph (DAG), each vertex corresponding to a group of processors that exists during some point of the computation; a directed edge is placed from group $G$ to group $G^{\prime}$ if $G \cap G^{\prime} \neq \emptyset$ and $G^{\prime}$ was formed by a reconfiguration involving processors in $G$ (this is discussed and formally defined in section 2). We say that two groups $G$ and $G^{\prime}$ are independent if there is no directed path connecting one to the other. For such a pattern $C$, the computation width of $C$, denoted $\mathbf{c w}(C)$, is the maximum number of independent groups reachable (along directed paths) in this DAG from any vertex. We show the following:

- (Upper bound.) For any computation pattern $C$, the randomized algorithm RS discussed above is $(1+\mathbf{c w}(C) / e)$-work competitive.

- (Lower bound.) For any scheduling algorithm $A(p, t)$, any $\epsilon>0$, and any 
nonzero $k \in \mathbb{N}$, there exist $p, t$, and a computation pattern $C$ so that $\mathbf{c w}(C)=$ $k$ and the work performed by algorithm $A(p, t)$ is at least $(1+k / e-\epsilon)$ times that of the off-line algorithm.

In particular, RS achieves the optimal competitive ratio over the set of all computation patterns with a given computation width.

Prior and related work; motivation. The problem of distributed cooperation for message-passing models was introduced and studied by Dwork, Halpern, and Waarts [11], who defined the notion of (task-oriented) work. The current problem of cooperation in partitionable networks has been the subject of active research. However, known solutions address narrow special cases, or provide substantially weaker bounds. Dolev, Segala, and Shvartsman [10] performed the first study of the problem in the partitionable setting. They model reconfiguration patterns for which the termination time of any on-line task-performing algorithm is greater than the termination time of an off-line task-performing algorithm by a factor linear in $p$. Malewicz, Russell, and Shvartsman [20] introduced the notion of $h$-waste that measures the worst-case redundant work performed by $h$ groups (or processors) when started in isolation and merged into a single group at some later time. While these results are deterministic, they only adequately describe such computation to the point of the first reconfiguration, where the reconfiguration is further assumed to simply merge groups together. Georgiou and Shvartsman [16] give upper bounds on work for an algorithm that performs work in the presence of network fragmentations and merges (i.e., limited patterns of reconfigurations) using a group communication service where processors initially start in a single group. They establish an upper bound of $O(\min (t \cdot p, t+t \cdot g(C)))$, where $g(C)$ is the total number of new groups formed during the computation pattern $C$. Note that $\mathbf{c w}(C) \leq g(C)$, and there can be an arbitrary gap between $\mathbf{c w}(C)$ and $g(C)$.

Thus prior work established reasonably tight (in the length of the processor schedule) results for a single first merge [20], illustrated the fact that on-line algorithms subject to diverging reconfiguration patterns incur linear (in $p$ ) overhead relative to an off-line algorithm [10], and showed an upper bound for an algorithm using group communication services for a limited pattern of reconfigurations starting with a single group [16].

The problem of cooperation on a common set of tasks in distributed settings has been studied in message-passing models $[7,8,11]$. These studies present various loadbalancing techniques for structuring the work for computing devices that are able to communicate by means of point-to-point messages. The studies of Georgiades, Mavronicolas, and Spirakis [13] and Papadimitriou and Yannakakis [22] investigated the impact of communication topology on the effectiveness of load-balancing.

The notion of competitiveness was introduced by Sleator and Tarjan [26] (see also Bartal, Fiat, and Rabani [5], Awerbuch, Kutten, and Peleg [3], and Ajtai et al. [1]).

Group communication services have become important as building blocks for fault-tolerant distributed systems. Such services enable processors located in a failureprone network to operate collectively as a group, using the services to multicast messages to group members (see the special issue [23]). To evaluate the effectiveness of partitionable group communication services, Sussman and Marzullo [27] proposed a measure (cushion) precipitated by a simple partition-aware application. Babaoglu et al. [4] studied systematic support for partition awareness based on group communication services in a wide range of application areas. As we mentioned earlier, cooperation on a common set of tasks has also been studied for algorithms using group 
communications $[10,16]$.

A related problem, referred to as Write-All, has been studied in the sharedmemory model. Early work in this area was reported by Kanellakis and Shvartsman [18], Martel and Subramonian [21], Kedem, Palem, and Spirakis [19], and Anderson and Woll [2]. In this setting the processors cooperate on updating locations in shared memory. The algorithmic techniques and analysis found there are quite different from the ones we present in this paper. Another related shared-memory problem, called Collect, requires that each processor learn the private values of all other processors. This problem was introduced by Shavit [25] and studied by Saks, Shavit, and Woll [24].

The structure of this paper is as follows. In section 2 we define the problem and model of computation. In section 3 we present and analyze the randomized algorithm RS. In section 4 we prove a lower bound for the problem. We conclude in section 5 .

Abstracts describing preliminary versions of the results in this paper appear in [14, $15]$.

2. Model and definitions. We consider a distributed system consisting of $p$ asynchronous processors connected by communication links; each processor has a unique identifier from the set $[p]=\{1,2, \ldots, p\}$; the value $p$ is known to all processors. The problem is then defined in terms of $t$ tasks with unique identifiers, initially known to all processors. The tasks are independent and idempotent-multiple executions of the same task have the same effect as a single execution. Processors may cease executing tasks only when they know the results of all tasks. This general problem is often referred to as Do-All.

The model is complicated by subjecting the processors to dynamic changes in the communication medium. In particular, at each instant of time, the network is partitioned into a collection of groups. Communication between processors in the same group is instantaneous and reliable, so that grouped processors may perfectly cooperate to complete tasks; communication across groups, however, is not possible. We consider the dynamic case where communication can be arbitrarily lost and reestablished. In particular, the computation of the processors is punctuated by a sequence of reconfigurations; each reconfiguration may induce an arbitrary change in the partition of the processors into groups. We shall assume that task executions are atomic with respect to reconfigurations. That is, a reconfiguration does not occur when some tasks are "halfway" through execution.

In order to focus on scheduling issues, we assume that processors in a single group work as a single virtual unit; indeed, we will treat them as a single asynchronous processor. In particular, upon the establishment of a new group by a reconfiguration, the processors in the group share their knowledge (of completed tasks) before they continue processing. A deterministic algorithm $D$ in this model is a rule which, given a processor (or group of processors) and a collection of tasks known to be completed, determines the next task for this processor (or group) to complete. Specifically, an algorithm is a function $D: 2^{[p]} \times 2^{[t]} \rightarrow[t]$; we note that the lower bounds proved in this paper actually apply to a wider class of algorithms that may in fact take into account the entire history of the computation of the group in question. For simplicity, we assume that $\forall P \subset[p], \forall T \subsetneq[t], D(P, T) \notin T$, which is to say that the algorithm never chooses to complete a task it already knows to be completed. Our goal will be to design algorithms that schedule the execution of the tasks to minimize the total work, where work is defined to be the number of tasks executed by all the processors during the entire computation (counting multiplicities). Ideally, the sets of tasks completed 
by two groups of processors when these groups are merged should be disjoint to avoid wasted effort. This is impossible in general, as processors must schedule their work in ignorance of future reconfigurations and, moreover, circumstances where two groups of processors merge that have collectively completed more than $t$ tasks will necessitate wasted work. A processor may cease executing tasks only when it knows the results of all tasks. We refer to this version of the Do-All problem as Omni-Do.

We will consider the behavior of an algorithm in the face of an adversary (which is oblivious in the sense of [6]) that determines both the sequence of reconfigurations and the number of tasks completed by each group before it is involved in another reconfiguration. Taken together, this information determines a computation pattern: this is a DAG, each vertex of which corresponds to a group $G$ of processors that existed during the computation; a directed edge is placed from $G_{1}$ to $G_{2}$ if $G_{2}$ was created by a reconfiguration involving $G_{1}$. We label each vertex of the DAG with the group of processors associated with that vertex and the total number of tasks that the adversary allows the group of processors to perform before the next reconfiguration occurs. As mentioned before, different adversaries (causing different sequences of reconfigurations) may give rise to the same computation pattern; the work caused by an adversary, however, depends only on the computation pattern determined by that adversary.

Specifically, if $t$ is the number of tasks and $p$ the number of processors, then such a computation pattern is a labeled and weighted DAG, which we call a $(p, t)$-DAG.

DEFINITION 2.1. A $(p, t)-D A G$ is a $D A G C=(V, E)$ augmented with a weight function $h: V \rightarrow[t] \cup\{0\}$ and a labeling $g: V \rightarrow 2^{[p]} \backslash\{\emptyset\}$ so that the following hold.

- For any maximal path $P=\left(v_{1}, \ldots, v_{k}\right)$ in $C, \sum h\left(v_{i}\right) \geq t$. (This guarantees that any algorithm terminates during the computation described by the DAG.) - $g$ possesses the following "initial conditions":

$$
[p]=\bigcup_{v: \text { in }(v)=0} g(v)
$$

- $g$ respects the following "conservation law":

there is a function $\phi: E \rightarrow 2^{[p]} \backslash\{\emptyset\}$ so that for each $v \in V$ with $\operatorname{in}(v)>0$,

$$
g(v)=\bigcup_{(u, v) \in E} \phi((u, v))
$$

and for each $v \in V$ with $\operatorname{out}(v)>0$,

$$
g(v)=\bigcup_{(v, u) \in E} \phi((v, u)) .
$$

In the above definition, $\dot{U}$ denotes disjoint union, and $\operatorname{in}(v)$ and $\operatorname{out}(v)$ denote the in-degree and out-degree of $v$, respectively. Finally, for two vertices $u, v \in V$, we write $u \leq v$ if there is a directed path from $u$ to $v$; we then write $u<v$ if $u \leq v$ and $u$ and $v$ are distinct.

Example. As an example, consider the (12,t)-DAG shown on Figure 2.1. Here we have $g_{1}=\left\{p_{1}\right\}, g_{2}=\left\{p_{2}, p_{3}, p_{4}\right\}, g_{3}=\left\{p_{5}, p_{6}\right\}, g_{4}=\left\{p_{7}\right\}, g_{5}=\left\{p_{8}, p_{9}, p_{10}, p_{11}, p_{12}\right\}$, $g_{6}=\left\{p_{1}, p_{2}, p_{3}, p_{4}, p_{6}\right\}, g_{7}=\left\{p_{8}, p_{10}\right\}, g_{8}=\left\{p_{9}, p_{11}, p_{12}\right\}, g_{9}=\left\{p_{1}, p_{2}, p_{3}, p_{4}\right.$, $\left.p_{6}, p_{8}, p_{10}\right\}, g_{10}=\left\{p_{5}, p_{11}\right\}$, and $g_{11}=\left\{p_{9}, p_{12}\right\}$.

This computation pattern models all asynchronous computations (adversaries) with the following behavior: (i) The processors in groups $g_{1}$ and $g_{2}$ and processor $p_{6}$ of group $g_{3}$ are regrouped during some reconfiguration to form group $g_{6}$. Processor $p_{5}$ of 


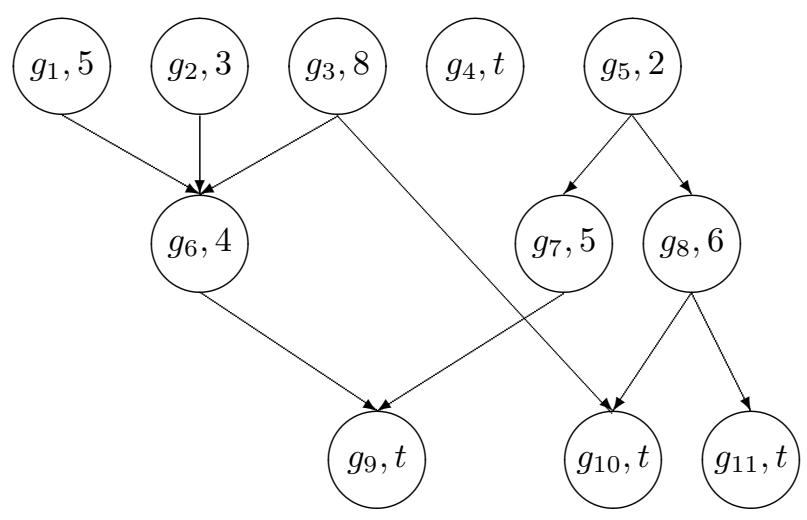

FIG. 2.1. An example of a $(12, t)-D A G$.

group $g_{3}$ becomes a member of group $g_{10}$ during the same reconfiguration (see below). Prior to this reconfiguration, processor $p_{1}$ (the singleton group $g_{1}$ ) has performed exactly 5 tasks, the processors in $g_{2}$ have cooperatively performed exactly 3 tasks, and the processors in $g_{3}$ have cooperatively performed exactly 8 tasks (assuming that $t>8$ ). (ii) Group $g_{5}$ is partitioned during some reconfiguration into two new groups, $g_{7}$ and $g_{8}$. Prior to this reconfiguration, the processors in $g_{5}$ have performed exactly 2 tasks. (iii) Groups $g_{6}$ and $g_{7}$ merge during some reconfiguration and form group $g_{9}$. Prior to this merge, the processors in $g_{6}$ have performed exactly 4 tasks (counting only the ones performed after the formation of $g_{6}$ and assuming that there are at least 4 tasks remaining to be done) and the processors in $g_{7}$ have performed exactly 5 tasks. (iv) The processors in group $g_{8}$ and processor $p_{5}$ of group $g_{3}$ are regrouped during some reconfiguration into groups $g_{10}$ and $g_{11}$. Prior to this reconfiguration, the processors in group $g_{8}$ have performed exactly 6 tasks (assuming that there are at least 6 tasks remaining, otherwise they would have performed the remaining tasks). (v) The processors in $g_{9}, g_{10}$, and $g_{11}$ run until completion with no further reconfigurations. (vi) Processor $p_{7}$ (the singleton group $g_{4}$ ) runs in isolation for the entire computation.

Let $D$ be a deterministic algorithm for Omni-Do and $C$ a computation pattern. We then let $W_{D}(C)$ denote the total work expended by algorithm $D$, where reconfigurations are determined according to the computation pattern $C . W_{D}$ is formally defined as follows.

Definition 2.2. Let $C$ be a $(p, t)-D A G$ and $D$ a deterministic algorithm for Omni-Do. $\boldsymbol{W}_{\boldsymbol{D}}(\boldsymbol{C})$ is defined inductively as follows.

- For a vertex $v$ of $C$ with $\operatorname{in}(v)=0$, define $L_{v}$ to be the set containing the first $h(v)$ tasks completed by group $g(v)$ according to $D$.

- Otherwise, $\operatorname{in}(v)>0$; in this case, let $\breve{L}_{v}=\bigcup_{u<v} L_{u}$ denote the collection of all tasks known to be complete at the inception of group $g(v)$. Then let $L_{v}$ be the first $h(v)$ tasks completed by group $g(v)$ according to $D$ starting with knowledge $\check{L}_{v}$. If $h(v)>t-\left|\check{L}_{v}\right|$, define $L_{v}=[t] \backslash \check{L}_{v}$.

Then $W_{D}(C)=\sum_{v \in C}\left|L_{v}\right|$.

We treat randomized algorithms as distributions over deterministic algorithms; for a set $\Omega$ and a family of deterministic algorithms $\left\{D_{r} \mid r \in \Omega\right\}$ we let $R=\mathcal{R}\left(\left\{D_{r} \mid\right.\right.$ $r \in \Omega\}$ ) denote the randomized algorithm where $r$ is selected uniformly at random from $\Omega$ and scheduling is done according to $D_{r}$. For a real-valued random variable $X$, we let $\mathbb{E}[X]$ denote its expected value. We let OPT denote the optimal (off-line) 
algorithm. Specifically, for each $C$ we define $W_{\mathrm{OPT}}(C)=\min _{D} W_{D}(C)$.

Definition 2.3 (see $[26,12,6]$ ). Let $\alpha$ be a real-valued function defined on the set of all $(p, t)-D A G s(\forall p$ and $t)$. A randomized algorithm $R$ is $\alpha$-competitive if for all computation patterns $C$,

$$
\mathbb{E}\left[W_{D_{r}}(C)\right] \leq \alpha(C) W_{O P T}(C),
$$

this expectation being taken over uniform choice of $r \in \Omega$.

Presently, we will introduce a function $\alpha$ that depends on a certain parameter (see Definition 2.7) of the graph structure of $C$. We note that, by definition, $\alpha \geq 1$.

We pause to develop some terminology that we will use in the rest of the paper.

Definition 2.4. A partially ordered set, or poset, is a pair $(P, \leq)$, where $P$ is a set and $\leq$ is a binary relation on $P$ for which (i) $\forall x \in P, x \leq x$; (ii) if $x \leq y$ and $y \leq x$, then $x=y$; and (iii) if $x \leq y$ and $y \leq z$, then $x \leq z$. For a poset $(P, \leq)$ we overload the symbol $P$, letting it denote both the set and the poset.

Definition 2.5. Let $P$ be a poset. We say that two elements $x$ and $y$ of $P$ are comparable if $x \leq y$ or $y \leq x$; otherwise $x$ and $y$ are incomparable. $A$ chain is a subset $H$ of $P$ such that any two elements of $H$ are comparable. An antichain is a subset $A$ of $P$ such that any two distinct elements of $A$ are incomparable. The width of $P$, denoted $\mathbf{w}(P)$, is the size of the largest antichain of $P$.

Associated with any DAG $C=(V, E)$ is the natural vertex poset $(V, \leq)$, where $u \leq v$ if and only if there is a directed path from $u$ to $v$. Then the width of $C$, denoted $\mathbf{w}(C)$, is the width of the poset $(V, \leq)$.

Definition 2.6. Given a $D A G C=(V, E)$ and a vertex $v \in V$, we define the predecessor graph at $v$, denoted $P_{C}(v)$ (or $P(v)$ when $C$ is implied), to be the subgraph of $C$ that is formed by the union of all paths in $C$ terminating at $v$. Likewise, the successor graph at $v$, denoted $S_{C}(v)$ (or $S(v)$ when $C$ is implied), is the subgraph of $C$ that is formed by the union of all the paths in $C$ originating at $v$.

Definition 2.7. The computation width of a $D A G C=(V, E)$, denoted $\mathbf{c w}(C)$, is defined as

$$
\mathbf{c w}(C)=\max _{v \in V} \mathbf{w}(S(v)) .
$$

Note that the processors that comprise a group formed during a computation pattern $C$ may be involved in many different groups at later stages of the computation, but no more than $\mathbf{c w}(C)$ of these groups will be forced to compute in ignorance of each other's progress.

In the $(12, t)$-DAG of Figure 2.1, the maximum width among all successor graphs is 3: $\mathbf{w}\left(S\left(\left(g_{5}, 2\right)\right)\right)=3$. Hence, the computational width of this DAG is 3 . Note that the width of the DAG is 6 (nodes $\left(g_{1}, 5\right),\left(g_{2}, 3\right),\left(g_{3}, 8\right),\left(g_{4}, t\right),\left(g_{7}, 5\right)$, and $\left(g_{8}, 6\right)$ form an antichain of maximum size).

3. Algorithm RS and its analysis. In this section we present the RANDOM SELECT (RS) algorithm and its analysis.

3.1. Description of algorithm RS. We consider the natural randomized algorithm RS, where a processor (or group), with knowledge that the tasks in a set $K \subset[t]$ have been completed, selects to next complete a task at random from the set $[t] \backslash K$. More formally, let $\Pi=\left(\pi_{1}, \ldots, \pi_{p}\right)$ be a $p$-tuple of permutations, where each $\pi_{i}$ is a permutation of $[t]$. We describe a deterministic algorithm $D_{\Pi}$ so that

$$
\mathrm{RS}=\mathcal{R}\left(\left\{D_{\Pi} \mid \Pi \in\left(S_{t}\right)^{p}\right\}\right) ;
$$


here $S_{t}$ is the collection of permutations on [t]. Let $G$ be a group of processors and $\gamma \in G$ the processor in $G$ with the lowest processor identifier. Then the deterministic algorithm $D_{\Pi}$ specifies that the group $G$, should it know that the tasks in $K \subset[t]$ have been completed, next completes the first task in the sequence $\pi_{\gamma}(1), \ldots, \pi_{\gamma}(t)$ which is not in $K$.

3.2. Analysis of algorithm RS. We now analyze the competitive ratio (in terms of work) of algorithm RS. We write $W_{\mathrm{RS}}(C)=\mathbb{E}\left[W_{\mathrm{RS}}(C)\right]$, this expectation taken over the random choices of the algorithm. Where $C$ can be inferred from context, we simply write $W_{\mathrm{RS}}$ and $W_{\mathrm{OPT}}$.

We first recall Dilworth's lemma [9], a duality theorem for posets.

Lemma 3.1 (see [9]). The width of a poset $P$ is equal to the minimum number of chains needed to cover $P$. (A family of nonempty subsets of a given set $S$ is said to cover $S$ if their union is $S$.)

We will also use a generalized degree-counting argument.

Lemma 3.2. Let $G=(U, V, E)$ be an undirected bipartite graph with no isolated vertices and $h: V \rightarrow \mathbb{R}$ a nonnegative weight function on $G$. For a vertex $v$, let $\Gamma(v)$ denote the vertices adjacent to $v$. Suppose that for some $A>0$ and for every vertex $u \in U$ we have $\sum_{v \in \Gamma(u)} h(v) \leq A$ and that for some $B>0$ and for every vertex $v \in V$ we have $\sum_{u \in \Gamma(v)} h(u) \geq B$. Then

$$
\frac{\sum_{u \in U} h(u)}{\sum_{v \in V} h(v)} \geq \frac{B}{A}
$$

Proof. We compute the quantity $\sum_{(u, v) \in E} h(u) h(v)$ by expanding according to each side of the bipartition:

$$
\begin{aligned}
A \sum_{u \in U} h(u) & \geq \sum_{u \in U}\left(h(u) \cdot \sum_{v \in \Gamma(u)} h(v)\right)=\sum_{(u, v) \in E} h(u) h(v) \\
& =\sum_{v \in V}\left(h(v) \cdot \sum_{u \in \Gamma(v)} h(u)\right) \geq B \sum_{v \in V} h(v) .
\end{aligned}
$$

As $A>0$ and $\sum_{v} h(v) \geq B>0$, we conclude that

$$
\frac{\sum_{u \in U} h(u)}{\sum_{v \in V} h(v)} \geq \frac{B}{A}
$$

as desired.

We now establish an upper bound on the competitive ratio of algorithm RS.

TheOREM 3.3. Algorithm $R S$ is $(1+\mathbf{c w}(C) / e)$-competitive for any $(p, t)$-DAG $C=(V, E)$.

Proof. Let $C$ be a $(p, t)$-DAG; recall that associated with $C$ are the two functions $h: V \rightarrow[t] \cup\{0\}$ and $g: V \rightarrow 2^{[p]} \backslash\{\emptyset\}$. For a subgraph $C^{\prime}=\left(V^{\prime}, E^{\prime}\right)$ of $C$, we let $H\left(C^{\prime}\right)=\sum_{v \in V^{\prime}} h(v)$. Recall that $P_{C}(v)$ and $S_{C}(v)$ denote the predecessor and successor graphs of $C$ at $v$. Then we say that a vertex $v \in V$ is saturated if $H\left(P_{C}(v)\right) \leq t$; otherwise, $v$ is unsaturated. Note that if $v$ is saturated, then the group $g(v)$ must complete $h(v)$ tasks regardless of the scheduling algorithm used. Along these same lines, if $v$ is an unsaturated vertex for which $t>\sum_{u<v} h(u)$, the group $g(v)$ must complete at least $\max \left(h(v), t-\sum_{u<v} h(u)\right)$ tasks under any scheduling algorithm. As these portions of $C$ which correspond to computation that must be performed by 
any algorithm will play a special role in the analysis, it will be convenient for us to rearrange the DAG so that all such work appears on saturated vertices. To achieve this, note that if $v$ is an unsaturated vertex for which $\sum_{u<v} h(u)<t$, we may replace $v$ with a pair of vertices, $v_{s}$ and $v_{u}$, where all edges directed into $v$ are redirected to $v_{s}$, all edges directed out of $v$ are changed to originate at $v_{u}$, the edge $\left(v_{s}, v_{u}\right)$ is added to $E$, and $h$ is redefined so that

$$
h\left(v_{s}\right)=t-\sum_{u<v} h(u) \quad \text { and } \quad h\left(v_{u}\right)=h(v)-h\left(v_{s}\right) .
$$

Note that the graph $C^{\prime}$ obtained by altering $C$ in this way corresponds to the same computation, in the sense that $W_{D}(C)=W_{D}\left(C^{\prime}\right)$ for any algorithm $D$. For the remainder of the proof we will assume that this alteration has been made at every relevant vertex, so that the graph $C$ satisfies the condition

$$
v \text { unsaturated } \Rightarrow \sum_{u<v} h(u) \geq t .
$$

Finally, for a vertex $v$, we let $T_{v}$ be the random variable equal to the number of tasks that RS completes at vertex $v$. Note that if $v$ is saturated, then $T_{v}=h(v)$. Let $\mathcal{S}$ and $\mathcal{U}$ denote the sets of saturated and unsaturated vertices, respectively. Given the above definitions, we immediately have

$$
W_{\mathrm{OPT}} \geq \sum_{s \in \mathcal{S}} h(s)
$$

and, by linearity of expectation,

$$
W_{\mathrm{RS}}=\mathbb{E}\left[\sum_{v} T_{v}\right]=\sum_{s \in \mathcal{S}} h(s)+\sum_{u \in \mathcal{U}} \mathbb{E}\left[T_{u}\right] \leq W_{\mathrm{OPT}}+\sum_{u \in \mathcal{U}} \mathbb{E}\left[T_{u}\right] .
$$

Our goal is to conclude that for some appropriate $\beta$,

$$
\mathbb{E}\left[\sum_{u \in \mathcal{U}} T_{u}\right] \leq \beta \cdot \sum_{s \in \mathcal{S}} h(s) \leq \beta \cdot W_{\mathrm{OPT}}
$$

and hence that RS is $1+\beta$ competitive. We will obtain such a bound by applying Lemma 3.2 to an appropriate bipartite graph, constructed next.

Given $C=(V, E)$, construct the (undirected) bipartite graph $G=\left(\mathcal{S}, \mathcal{U}, E_{G}\right)$, where $E_{G}=\{(s, u) \mid s<u\}$. As in Lemma 3.2, for a vertex $v$, we let $\Gamma(v)$ denote the set of vertices adjacent to $v$. Now assign weights to the vertices of $G$ according to the rule $h^{*}(v)=\mathbb{E}\left[T_{v}\right]$. Note that for $s \in \mathcal{S}, h^{*}(s)=h(s)$ and hence by condition (3.1) above, we immediately have the bound

$$
\forall u \in \mathcal{U}, \quad \sum_{s \in \Gamma(u)} h^{*}(s) \geq t
$$

We now show that $\forall s \in \mathcal{S}$,

$$
\sum_{u \in \Gamma(s)} h^{*}(u) \leq \mathbf{c w}(C) \cdot \frac{t}{e}
$$


Before proceeding to establish this bound, note that (3.3) and (3.4), together with Lemma 3.2, imply that

$$
\begin{aligned}
W_{\mathrm{RS}}(C) & \leq \sum_{s \in \mathcal{S}} h(s)+\sum_{u \in \mathcal{U}} h^{*}(u) \leq\left(1+\frac{\mathbf{c w}(C)}{e}\right) \sum_{s \in \mathcal{S}} h(s) \\
& \leq\left(1+\frac{\mathbf{c w}(C)}{e}\right) W_{\mathrm{OPT}}(C),
\end{aligned}
$$

as desired.

Returning now to (3.4), let $s \in \mathcal{S}$ be a saturated vertex and consider the successor graph (of $C$ ) at $s, S_{C}(s)$. By Lemma 3.1 (Dilworth's lemma), there exist $w \triangleq \mathbf{w}\left(S_{C}(s)\right) \leq \mathbf{c w}(C)$ paths in $S_{C}(s), P_{1}, P_{2}, \ldots, P_{w}$, so that their union covers $S_{C}(s)$. Let $X_{i}$ be the random variable whose value is the number of tasks performed by RS on the portion of the path $P_{i}$ consisting of unsaturated vertices. Note that if $u \in V$ is unsaturated and $u \leq v$, then $v$ is unsaturated and hence, for each path $P_{i}$, there is a first unsaturated vertex $u_{i}^{0}$ after which every vertex of $P_{i}$ is unsaturated. Note now that for a fixed individual task $\tau$, conditioned upon the event that $\tau$ is not yet complete, the probability that $\tau$ is not chosen by RS for completion at a given selection point in $P_{C}\left(u_{i}^{0}\right)$ is no more than $(1-1 / t)$. Let $L_{i}$ be the random variable whose value is the set of tasks left incomplete by RS at the formation of the group $g\left(u_{i}^{0}\right)$. As $u_{i}^{0}$ is unsaturated, $\sum_{v<u_{i}^{0}} h(v) \geq t$ by condition (3.1) and hence, for each $i$,

$$
\operatorname{Pr}\left[\tau \in L_{i}\right] \leq(1-1 / t)^{t} \leq 1 / e .
$$

As there are a total of $t$ tasks,

$$
\mathbb{E}\left[\left|L_{i}\right|\right] \leq t / e
$$

Of course, since RS completes a new task at each step, $X_{i} \leq\left|L_{i}\right|$ so that $\mathbb{E}\left[X_{i}\right] \leq t / e$, and by linearity of expectation

$$
\mathbb{E}\left[\sum_{i} X_{i}\right] \leq w \cdot t / e
$$

Now every unsaturated vertex in $S_{C}(s)$ appears in some $P_{i}$ and hence

$$
\sum_{u \in \Gamma(s)} h^{*}(u) \leq \mathbb{E}\left[\sum_{i} X_{i}\right] \leq w t / e \leq \mathbf{c w}(C) \cdot t / e,
$$

as desired.

Theorem 3.3 implies a constant upper bound for patterns that consist entirely of merges (that is, where all reconfigurations are given by taking unions of existing groups). This subsumes the results reported in [14].

Corollary 3.4. Algorithm $R S$ is $\left(1+\frac{1}{e}\right)$-competitive for any $(p, t)$-DAGC with $\mathbf{c w}(C)=1$.

Remark. The proof of Theorem 3.3 can be slightly modified to yield an interesting result for deterministic scheduling algorithms. Let $D$ be a deterministic scheduling algorithm for Omni-Do. In the proof of Theorem 3.3, $h^{*}(v)$ was defined as the expected number of tasks performed by algorithm RS at node $v$. For algorithm $D$, if we define $h^{*}(v)$ to be the actual number of tasks performed by the algorithm at node $v$, then it is not difficult to see that (3.4) becomes $\sum_{u \in \Gamma(s)} h^{*}(u) \leq \mathbf{c w}(C) \cdot t$ (provided that no processor in $D$ performs a task that already knows its result). This leads to the conclusion that any (nontrivial) deterministic algorithm for Omni-Do is $(1+\mathbf{c w}(C))$-competitive for any computation pattern $C$. 
4. A lower bound. We begin with a lower bound for deterministic algorithms. This is then applied to give a lower bound for randomized algorithms in Corollary 4.2.

THEOREM 4.1. Let $a: \mathbb{N} \rightarrow \mathbb{R}$ and $D$ be a deterministic scheduling algorithm for Omni-Do so that $D$ is a $(\mathbf{c w}(\cdot))$-competitive (that is, $D$ is $\alpha$-competitive, for a function $\alpha=a \circ \mathbf{c w})$ ). Then $a(c) \geq 1+c / e$.

Proof. Fix $k \in \mathbb{N}$. Consider the case when $t=p=g \gg k$ and $t \bmod k=0, g$ being the number of initial groups. We consider a computation pattern $C_{\mathbf{G}}$ determined by a tuple $\mathbf{G}=\left(G_{1}, \ldots, G_{t / k}\right)$, where each $G_{i} \subset[t]$ is a set of size $k$ and $\bigcup_{i} G_{i}=[t]$. Initially, the computation pattern $C_{\mathbf{G}}$ has the processors synchronously proceed until each has completed $t / k$ tasks; at this point, the processors in $G_{i}$ are merged and allowed to exchange information about task executions. Each $G_{i}$ is then immediately partitioned into $c$ groups (this establishes that the computation width is $c$ ). Note that the off-line optimal algorithm accrues exactly $t^{2} / k$ work for this computation history (it terminates prior to the partitions of the $G_{i}$ ).

We will show that for any scheduling deterministic Omni-Do algorithm $D$, there is a selection of the $G_{i}$ so that

$$
W_{D}\left(C_{\mathbf{G}}\right) \geq t^{2} / k\left[1+c\left(1-\frac{1}{k}\right)^{k}-o(1)\right],
$$

and hence that $a(c) \geq 1+c / e$. Consider the behavior of $D$ when $\mathbf{G}$ is selected at random, uniformly among all such tuples. Let $P_{i} \subset[t]$ be the subset of $t / k$ tasks completed by processor $i$ before the merges take place; these sets are determined by the algorithm $D$. We begin by bounding

$$
\mathbb{E}_{\mathbf{G}}\left[\left|\bigcup_{i \in G_{1}} P_{i}\right|\right]
$$

To this end, consider an experiment where we select $k$ sets $Q_{1}, \ldots, Q_{k}$, each $Q_{i}$ selected independently and uniformly from the set $\left\{P_{i}\right\}$. Now, for a specific task $\tau$, let $p_{\tau}=$ $\operatorname{Pr}_{Q_{1}}\left[\tau \notin Q_{1}\right]$, so that $\operatorname{Pr}_{Q_{i}}\left[\tau \notin \bigcup_{i} Q_{i}\right]=p_{\tau}^{k}$. As the $Q_{i}$ are selected independently,

$$
\mathbb{E}_{Q_{i}}\left[\left|[t]-\bigcup_{i} Q_{i}\right|\right]=\sum_{\tau} p_{\tau}^{k}
$$

Observe now that

$$
\sum_{\tau}\left(1-p_{\tau}\right)=\sum_{\tau} \operatorname{Pr}_{Q_{1}}\left[\tau \in Q_{1}\right]=\mathbb{E}_{Q_{1}}\left[\left|Q_{1}\right|\right]=t / k
$$

and hence $\sum_{\tau} p_{\tau}=t(1-1 / k)$. As the function $x \mapsto x^{k}$ is convex on $[0, \infty), \sum_{\tau} p_{\tau}^{k}$ is minimized when the $p_{\tau}$ are equal, and we must have

$$
\mathbb{E}_{Q_{i}}\left[\left|[t]-\bigcup_{i} Q_{i}\right|\right] \geq t \cdot\left(1-\frac{1}{k}\right)^{k}
$$

Now observe that, conditioned on the $Q_{i}$ being distinct, the distribution of $\left(Q_{1}, \ldots, Q_{k}\right)$ is identical to that of $\left(P_{g_{1}^{1}}, \ldots, P_{g_{k}^{1}}\right)$, where the random variable $G_{1}=$ $\left\{g_{1}^{1}, \ldots, g_{k}^{1}\right\}$. Considering that $\operatorname{Pr}\left[\exists i \neq j, Q_{i}=Q_{j}\right] \leq k^{2} / t$, we have

$$
\mathbb{E}_{Q_{i}}\left[\left|[t]-\bigcup_{i} Q_{i}\right|\right] \leq\left(1-\frac{k^{2}}{t}\right) \mathbb{E}_{\mathbf{G}}\left[t-\left|\bigcup_{i \in G_{1}} P_{i}\right|\right]+1 \cdot \frac{k^{2}}{t},
$$


and hence as $t \rightarrow \infty$, we see that the expected number of tasks remaining for those processors in group $G_{1}$ is

$$
\mathbb{E}_{\mathbf{G}}\left[t-\left|\bigcup_{i \in G_{1}} P_{i}\right|\right] \geq t(1-1 / k)^{k}-o(1) .
$$

Of course, the distribution of each $G_{i}$ is the same, so that

$$
\mathbb{E}_{\mathbf{G}}\left[\sum_{i=1}^{t / k}\left(t-\left|\bigcup_{j \in G_{i}} P_{j}\right|\right)\right]=[1-o(1)]\left(\frac{t}{k}\right) \cdot t\left(1-\frac{1}{k}\right)^{k}
$$

In particular, there must exist a specific selection of $\mathbf{G}=\left(G_{1}, \ldots, G_{t / k}\right)$ which achieves this bound. Recall that every $G_{i}$ is partitioned into $c$ groups. Therefore, for such $\mathbf{G}$, the total work is at least

$$
\frac{t^{2}}{k} \cdot\left(1+[1-o(1)] \cdot c \cdot\left(1-\frac{1}{k}\right)^{k}\right) .
$$

As $\lim _{k \rightarrow \infty}\left(1-\frac{1}{k}\right)^{k}=\frac{1}{e}$, this completes the proof.

As the above stochastic computation pattern $C_{\mathbf{G}}$ is independent of the deterministic algorithm $D$, this immediately gives rise to a lower bound for randomized algorithms.

Corollary 4.2. Let $\mathcal{R}\left(\left\{D_{r} \mid r \in \Omega\right\}\right)$ be a randomized scheduling algorithm for Omni-Do that is $(a \circ \mathbf{c w})$-competitive. Then $a(c) \geq 1+c / e$.

Proof. Assume for contradiction that for some $c, a(c)<1+c / e$, and let $k$ be large enough so that $\left(1-\frac{1}{k}\right)^{k}>a(c)-1$. For this $k$ we proceed as in the proof above, considering a random $\mathbf{G}$ and the computation pattern $C_{\mathbf{G}}$ with $t=g=p$ congruent to $0 \bmod k, g$ being the number of initial groups. Then, as above,

$$
\begin{aligned}
\mathbb{E}_{\mathbf{G}}\left[\mathbb{E}_{r}\left[W_{D_{r}}\left(C_{\mathbf{G}}\right)\right]\right] & =\mathbb{E}_{r}\left[\mathbb{E}_{\mathbf{G}}\left[W_{D_{r}}\left(C_{\mathbf{G}}\right)\right]\right] \\
& \geq \min _{r}\left[\mathbb{E}_{\mathbf{G}}\left[W_{D_{r}}\left(C_{\mathbf{G}}\right)\right]\right] \\
& \geq \frac{t^{2}}{k} \cdot\left(1+[1-o(1)] \cdot c \cdot\left(1-\frac{1}{k}\right)^{k}\right) .
\end{aligned}
$$

Hence there exists a $\mathbf{G}$ so that $\mathbb{E}_{r}\left[W_{D_{r}}\left(C_{\mathbf{G}}\right)\right] \geq \frac{t^{2}}{k} \cdot\left(1+[1-o(1)] \frac{c}{e}\right)$, which completes the proof.

5. Conclusions and open problems. We established bounds on the competitive ratio of a natural randomized algorithm for scheduling in partitionable networks and show, furthermore, that for the relevant gradation of computation patterns these bounds are tight. We showed how to characterize algorithm competitiveness in terms of computation width, a precise property of a DAG that describes the computation history. These results lead to a better understanding of the effectiveness of computation in group communication schemes, a widely used paradigm for computing in distributed environments.

One outstanding open question is how to derandomize the schedules used by taskperforming algorithms in this work. Specifically, we would like to construct deterministic scheduling algorithms that are $(1+\mathbf{c w}(C) / e)$-competitive for any computation 
pattern $C$. Another promising direction is to study the task-performing paradigm in the models of computation that combine network reconfigurations with processor failures. The goal is to establish complexity results that show how performance of task-performing algorithms depends both on the extent of the network reconfiguration and on the number of processor failures.

\section{REFERENCES}

[1] M. Ajtai, J. Aspnes, C. Dwork, and O. WaArts, A theory of competitive analysis for distributed algorithms, in Proceedings of the 35th Symposium on Foundations of Computer Science (FOCS 1994), IEEE, Los Alamitos, CA, 1994, pp. 401-411.

[2] R. J. Anderson And H. Woll, Algorithms for the certified write-all problem, SIAM J. Comput., 26 (1997), pp. 1277-1283.

[3] B. Awerbuch, S. Kutten, And D. Peleg, Competitive distributed job scheduling, in Proceedings of the 24th ACM Symposium on Theory of Computing (STOC 1992), ACM, New York, 1992, pp. 571-580.

[4] O. Babaoglu, R. Davoli, A. Montresor, and R. Segala, System support for partitionaware network applications, in Proceedings of the 18th IEEE International Conference on Distributed Computing Systems (ICDCS 1998), IEEE, Los Alamitos, CA, 1998, pp. 184-191.

[5] Y. Bartal, A. Fiat, And Y. Rabani, Competitive algorithms for distributed data management, in Proceedings of the 24th ACM Symposium on Theory of Computing (STOC 1992), ACM, New York, 1992, pp. 39-50.

[6] S. Ben-David, A. Borodin, R. Karp, G. Tardos, and A. Wigderson, On the power of randomization in on-line algorithms, Algorithmica, 11 (1994), pp. 2-14.

[7] B. Chlebus, R. De Prisco, and A. A. Shvartsman, Performing tasks on restartable messagepassing processors, Distributed Comput., 14 (2001), pp. 49-64.

[8] R. De Prisco, A. Mayer, And M. Yung, Time-optimal message-efficient work performance in the presence of faults, in Proceedings of the 13th ACM Symposium on Principles of Distributed Computing (PODC 1994), ACM, New York, 1994, pp. 161-172.

[9] R. P. Dilworth, A decomposition theorem for partially ordered sets, Ann. of Math., 51 (1950), pp. 161-166.

[10] S. Dolev, R. Segala, and A. A. Shvartsman, Dynamic load balancing with group communication, in Proceedings of the 6th International Colloquium on Structural Information and Communication Complexity (SIROCCO 1999), Carleton Scientific, Waterloo, ON, Canada, 1999, pp. 111-125.

[11] C. Dwork, J. Y. Halpern, And O. WaArts, Performing work efficiently in the presence of faults, SIAM J. Comput., 27 (1998), pp. 1457-1491.

[12] A. Fiat, R. M. Karp, M. Luby, L. A. McGeoch, D. D. Sleator, and N. E. Young, Competitive paging algorithms, J. Algorithms, 12 (1991), pp. 685-699.

[13] S. Georgiades, M. Mavronicolas, and P. Spirakis, Optimal, distributed decision-making: The case of no communication, in Proceedings of the 12th International Symposium on Fundamentals of Computation Theory (FCT 1999), Lecture Notes in Comput. Sci. 1684, Springer-Verlag, Berlin, 1999, pp. 293-303.

[14] Ch. Georgiou, A. Russell, and A. A. Shvartsman, Optimally work-competitive scheduling for cooperative computing with merging groups (brief announc.), in Proceedings of the 22nd ACM Symposium on Principles of Distributed Computing (PODC 2002), ACM, New York, 2002, p. 132.

[15] Ch. Georgiou, A. Russell, and A. A. Shvartsman, Work-competitive scheduling for cooperative computing with dynamic groups, in Proceedings of the 35th Annual ACM Symposium on Theory of Computing (STOC 2003), ACM, New York, 2003, pp. 251-258.

[16] Ch. Georgiou and A. A. Shvartsman, Cooperative computing with fragmentable and mergeable groups, J. Discrete Algorithms, 1 (2003), pp. 211-235.

[17] J. F. Groote, W. H. Hesselink, S. Mauw, and R. Vermeulen, An algorithm for the asynchronous Write-All problem based on process collision, Distributed Comput., 14 (2001), pp. $75-81$.

[18] P. C. Kanellakis And A. A. Shvartsman, Fault-Tolerant Parallel Computation, Kluwer Academic, Dordrecht, The Netherlands, 1997.

[19] Z. M. Kedem, K. V. Palem, and P. Spirakis, Efficient robust parallel computations, in Proceedings of the 22nd ACM Symposium on Theory of Computing (STOC 1990), ACM, New 
York, 1990, pp. 138-148.

[20] G. Malewicz, A. Russell, and A. A. Shvartsman, Distributed cooperation during the absence of communication, in Proceedings of the 14th International Symposium on Distributed Computing (DISC 2000), Lecture Notes in Comput. Sci. 1914, Springer-Verlag, Berlin, 2000, pp. 119-133.

[21] C. Martel and R. Subramonian, On the complexity of certified Write-All algorithms, J. Algorithms, 16 (1994), pp. 361-387.

[22] C. H. Papadimitriou And M. Yannakakis, On the value of information in distributed decisionmaking, in Proceedings of the 10th ACM Symposium on Principles of Distributed Computing (PODC 1991), ACM, New York, 1991, pp. 61-64.

[23] D. Powell, Ed., Special Issue on Group Communication Services, Comm. ACM, 39 (1996).

[24] M. SaKs, N. Shavit, AND H. Woll, Optimal time randomized consensus-making resilient algorithms fast in practice, in Proceedings of the 2nd ACM-SIAM Symposium on Discrete Algorithms (SODA 1991), ACM, New York, 1991, pp. 351-362.

[25] N. Shavit, Concurrent Timestamping, Ph.D. thesis, The Hebrew University, 1989.

[26] D. Sleator and R. Tarjan, Amortized efficiency of list update and paging rules, Comm. ACM, 28 (1985), pp. 202-208.

[27] J. B. Sussman And K. Marzullo, The Bancomat problem: An example of resource allocation in a partitionable asynchronous system, in Proceedings of the 12th International Symposium on Distributed Computing (DISC 1998), Lecture Notes in Comput. Sci. 1499, Springer-Verlag, Berlin, 1998, pp. 363-377. 